\title{
A INFLUÊNCIA DOS FATORES CLIMÁTICOS NOS CASOS DE DENGUEDAS 10 CIDADES MAIS POPULOSAS DO MATO GROSSO DO SUL EM 2009
}

\author{
Bruna dos Santos Silva ${ }^{(a)}$, Charlei Aparecido da Silva ${ }^{(b)}$ \\ ${ }^{(a)}$ Acadêmica do programa de pós-graduação em Geografia, Universidade Federal de Grande Dourados, \\ bruna.inpe@gmail.com \\ ${ }^{(b)}$ Docente do programa de pós-graduação em Geografia, Universidade Federal de Grande Dourados, \\ charleisilva@ufgd.edu.br
}

Eixo: 3. Climatologia em diferentes níveis escalares: mudanças e variabilidades

\begin{abstract}
Resumo
O objetivo desse trabalho constituiu-se em analisar a manifestação da dengue nas 10 maiores cidades do Mato Grosso Sul no ano de 2009, relacionando a quantidade de casos com a temperatura média e pluviosidade total. Confeccionaram gráficos e estabeleceu-se correlações estatísticas entre as variáveis. O ano de 2009 foi escolhido devido ao grande surto de casos que o estado notificou. Após a analise, observou-se resultados interessantes como o caso da cidade de Corumbá, que encontra-se na fronteira com a Bolívia e teve o maior número de casos notificados no ano, porém sua correlação com os dados pluviométricos foram considerados perfeitamente negativos.
\end{abstract}

Palavras chave:Saúde e Dengue, Climatologia Geográfica, Mato Grosso Sul, Epidemia

\section{Introdução}

A dengue é uma arbovirose transmitida pela picada do mosquito fêmea da espécie Aedes aegypti infectada, e sua forma mais grave é a dengue hemorrágica. Os diferentes sorotipos (DEN 1, 2, 3 e 4) permitiram sua grande expansão no mundo, sendo considerado um dos principais problemas de saúde pública, gerando gastos de milhões de dólares anualmente. A dengue clássica registra de 50 a 100 milhões de casos/ano no mundo e cerca de 3 bilhões de pessoas estão vulneráveis ao vírus. O número de internações por febre hemorrágica chega a 500 mil com taxa mortalidade de 5\%, por consequências da patologia (MONDINI\& CHIARAVALLOTI NETO, 2007).

O vírus da dengue persiste na natureza mediante o ciclo de transmissão homem- mosquitohomem. A transmissão se faz pela picada do mosquito fêmea infectado, porém, a presença desse mosquito não ocorre em todas regiões geográficas devido aos fatores limitantes ao seu desenvolvimento. 
As condições hidroclimáticas exercem grande influência sobre a distribuição geográfica dos seres vivos. Os limites das áreas de distribuição são muitas vezes determinados pela temperatura e pluviosidade, que agem como fator limitante (DAJOZ, 1983; ODUM, 1988).

A pluviosidade e principalmente a temperatura afetam significativamente na longevidade, fecundidade e atividade hematofágica do Aedes, como apontaram os estudos de Silva e Neves (1989) e Calado e Navarro- Silva (2002). Destaca-se que as baixas temperaturas geralmente são nocivas ao desenvolvimento do mosquito.

A temperatura favorável para o desenvolvimento do mosquito Aedes aegypti encontra-se entre $21^{\circ} \mathrm{C}$ e $29^{\circ} \mathrm{C}$, de acordo com Torres (1998) o mosquito não resiste à temperatura inferior a $6^{\circ} \mathrm{C} \mathrm{e}$ superiores a $42^{\circ} \mathrm{C}$.

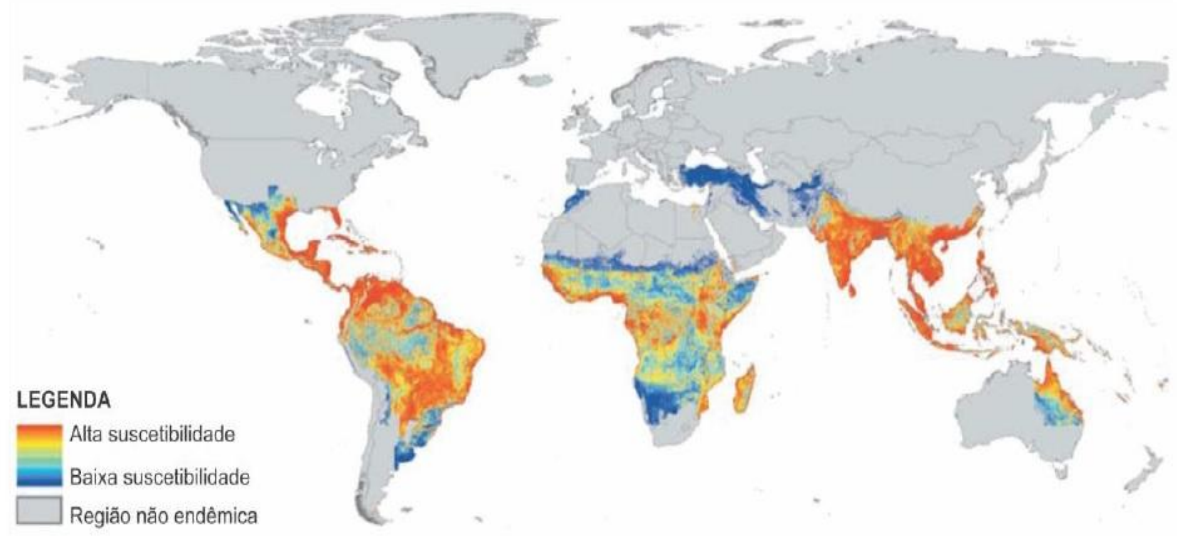

Figura 1:Risco global de contaminação da dengue

Fonte: Simmons et al., 2012.

Para Confalonieri (2003), a temperatura, a precipitação pluviométrica, a umidade relativa e o ciclo hidrológico, afetam a sobrevivência e reprodução de agentes patogênicos, principalmente dos vetores de agentes infecciosos, como os mosquitos transmissores da malária e da dengue. $\mathrm{O}$ mosquito transmissor da dengue, o Aedes aegypti distribui-se geograficamente entre os paralelos $45^{\circ}$ de latitude norte e $35^{\circ}$ de latitude sul, perfazendo uma área caracterizada por altas temperaturas e intensa pluviosidade em determinados períodos, além da umidade do ar, fatores favoráveis à proliferação do mosquito, segundo Costa (2001). Cada mosquito vive em média, 30 dias e, quando férteis, as fêmeas chegam a depositar entre 150 a 200 ovos (SILVA, 2007), sendo possível que uma única fêmea contaminada gere um surto da doença.

Na América Latina em especial, considerando-se a elevação das médias térmicas globais e das precipitações, estima-se que extensas áreas venham a sofrer devido à intensificação da 
disseminação de vetores de doenças como a malária e a dengue, possibilitando a expansão das áreas geográficas de transmissão de doenças (MENDONÇA, 2003, 2004 e 2006).

No Brasil, a dengue tornou-se um problema importante devido às dramáticas proporções que se têm tomado. Entre 1990 a 2007, quase 4,5 milhões de casos de dengue, enquanto que o número de municípios infestados dobrou nos últimos 10 anos. Entre os anos de 2000 a 2006, 77\% dos municípios apresentam casos autóctones.

\section{1- Clima e saúde}

Apesar das relações entre clima e saúde serem demasiadamente conhecidas, a definição de parâmetros dos efeitos de cada um dos componentes climáticos sobre as doenças é um esforço que muitos pesquisadores têm alcançando a partir de modelos variados.

A saúde humana é fortemente influenciada pelo clima através das condições térmicas, de dispersão (ventos e poluição) e de umidade do ar, exercendo destacada influência sobre a manifestação de muitas doenças, epidemias e endemias, criando condições favoráveis ao desenvolvimento dos transmissores de doenças contagiosas. (MENDONÇA, 2000).

Os principais fatores climáticos para dispersão de vetores e doenças são: temperatura, precipitação, umidade e velocidade do vento (CONSOLI, 1994).

O clima é um elemento importante para a saúde e a qualidade de vida. Entretanto, o impacto de determinado clima sobre o organismo não corresponde de maneira exata à percepção do prazer e conforto que ele proporciona; em alguns casos pode existir um grande hiato entre os mesmos. (BESANCENOT, 2001).

A concentração de chuvas aliadas a um ambiente com temperaturas ideais cria uma situação ótima para sua reprodução e dispersão do Aedes aegypti. Embora possa manter uma população considerável durante as estações menos chuvosas, a custa dos criadouros semipermanentes e independentes das chuvas (caixas d'água, cisternas, latões etc.), é durante a estação chuvosa que sua população realmente alcança níveis elevados e de importância para fins de transmissão de patógenos (CONSOLI, 1994).

A temperatura e a precipitação podem influenciar na transmissão da dengue, impactando a população do vetor. A abundância do vetor predominante (Aedes aegypti) é parcialmente regulada pela precipitação, criando focos de reprodução e estimulando o desenvolvimento dos ovos. Já a temperatura influencia na habilidade do mosquito para sobreviver e determina seu desenvolvimento e taxas reprodutivas (JOHANSSON, 2009).

MENDONÇA (2011) relatou que a situação epidemiológica como a dengue pode agravar-se devido à variabilidade climática observada nos últimos anos, com a possibilidade real da expansão das áreas geográficas de transmissão do vírus. 
Sendo assim, as interações entre clima e saúde devem levar em consideração não só fatores físicos como aspectos climáticos, mas também fatores sociais como a urbanização, que se tornam responsáveis pela construção de ambientes vulneráveis e consequentemente facilitadores de transmissão de doenças.

O foco dessa pesquisa, desenvolvida no Programa de Pós-Graduação em Geografia, junto ao Laboratório de Geografia Física, visa compreender a ocorrência e a distribuição da dengue no Mato Grosso do Sul no período de 2000 a 2015 e sua participação no total dos casos registrados no Brasil.

\section{1-2- O Clima do Mato Grosso do Sul}

Diversos fatores físico-geográficos e dinâmicos explicam o comportamento da temperatura no Estado de Mato Grosso do Sul. Se na primavera e no verão, o calor é quase uma constante, são comuns dias muito frios no inverno. Em Mato Grosso do Sul, durante o inverno, imediatamente após a passagem da frente polar e da chuva frontal, a umidade é muito grande, geralmente acima de 95\%. Dias depois, há rápida elevação das temperaturas, com forte redução da umidade, até a chegada de nova frente polar.

Segundo Zavattini, o estado do Mato Grosso do Sul, encontra-se na confluência dos principais sistemas atmosféricos da América do Sul, possuindo mais de um tipo de regime pluviométrico, havendo áreas com regime do tipo "Brasil Central" e outras com regime do tipo "Brasil Meridional".

$\mathrm{Na}$ primavera e no verão, as temperaturas mantêm-se elevadas quase que diariamente, em quase todo o Estado. As temperaturas mais elevadas do Estado de Mato Grosso do Sul registram-se no Pantanal Mato-grossense, vasta área de baixas altitudes e densa rede hidrográfica.

Na depressão da planície pantaneira, os índices da umidade relativa do ar são elevados durante o ano todo. No verão, com médias superiores a $85 \%$, o desconforto térmico é determinado mais pela excessiva quantidade de vapor d'água na atmosfera do que pela temperatura. Nos planaltos, a baixa umidade relativa do ar no inverno intensifica os efeitos da estiagem.

Outro aspecto do regime térmico a ser destacado é a variabilidade da temperatura em período máximo de 24 horas. As máximas temperaturas registradas nas horas do dia chegam a $30^{\circ} \mathrm{C}$ no aquecimento pré-frontal, caindo rapidamente, próximo ou abaixo de $0^{\circ} \mathrm{C}$, quando sob o domínio da massa polar.

De acordo com Parra (2010), o alinhamento topográfico, disposto no sentido longitudinal, exibe feições morfológicas bem definidas: a planície, a oeste; o planalto, a leste. Essa disposição exerce influência no comportamento da dinâmica atmosférica de Mato Grosso do Sul. Se por um lado a depressão da Planície do Pantanal funciona como corredor para a penetração das massas polares no inverno, por outro, é responsável pelas condições do tempo abafado no verão. 
Segundo Zavattini (2009), o estado do Mato Grosso do Sul possui quatro Unidades Climáticas (UC), influenciadas pelas condições de temperatura, altitude e pressão atmosférica, e nesse sentido a pesquisa visa e busca associar os casos de dengue a cada UC do Estado.

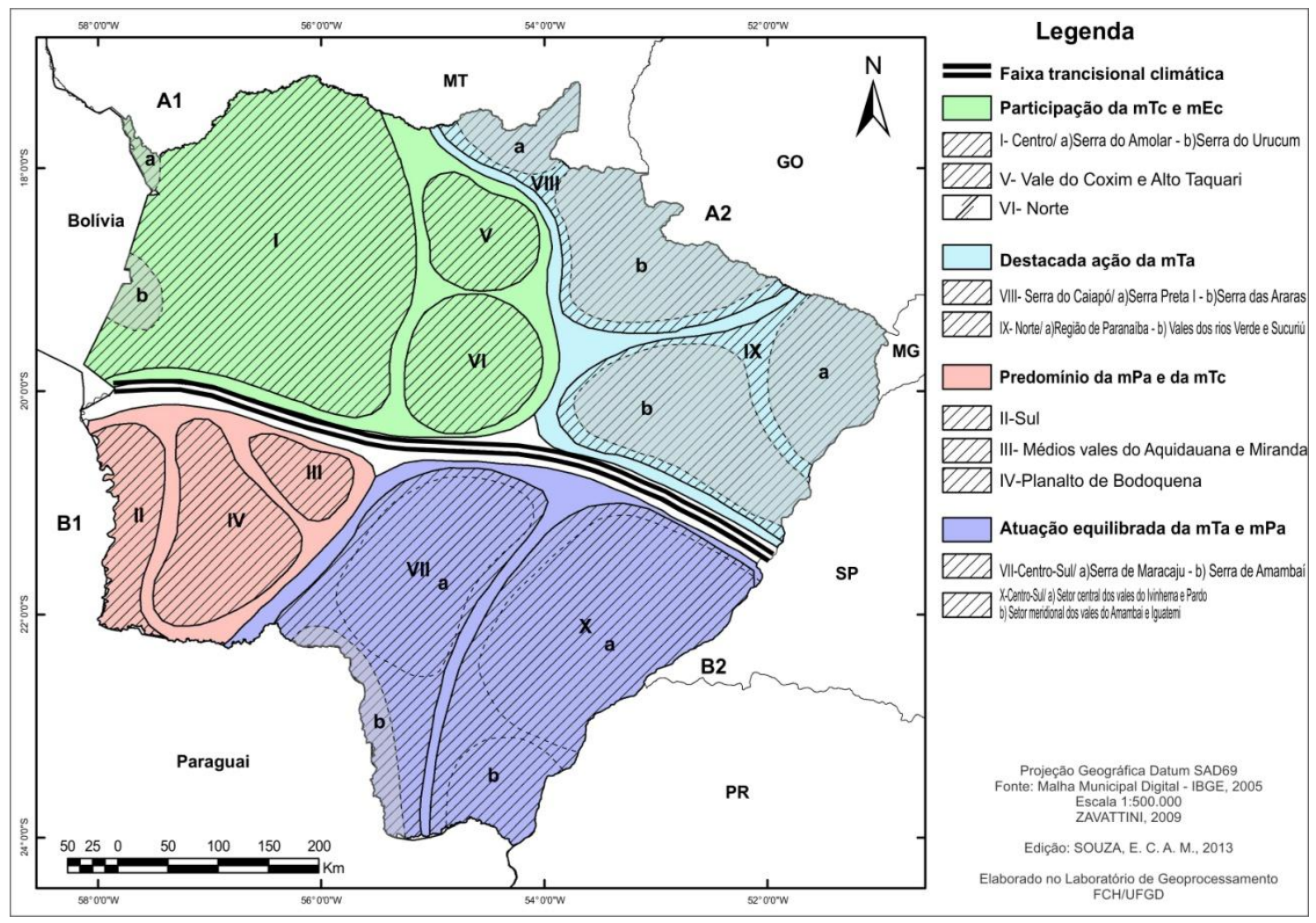

Figura 2: Mapa das Unidades Climáticas do Mato Grosso do Sul

\section{2 - Material e métodos.}

Mato Grosso do Sul é o $6^{\circ}$ Estado do país em extensão territorial com 35.158,7 km. Faz fronteira com 2 países- Paraguai e Bolívia, bem como se limita com os Estados de Goiás, Mato Grosso, Minas Gerais, Paraná e São Paulo. Está localizado ao sul da Região Centro-Oeste. Sua população estimada em 2015 é de 2.651 .235 habitantes. O Estado apresenta atualmente 78 municípios.

Ao trabalhar com variáveis climáticas, deve-se atentar para as diferenças conceituais, e de alcance temporal, entre variabilidade climática e mudanças mais estruturais no clima, chamadas de mudanças climáticas [Confalonieri, 2003; Kelly-Hope e Thomson, 2008]. A primeira normalmente é dominada por variações inter-anuais e pode ser entendida pela propriedade do sistema climático terrestre em oscilar os padrões climáticos nos níveis local, regional e global. Efeitos climáticos do fenômeno El Nino, por exemplo, podem ser endereçados a variabilidades climáticas pontuais. A mudança climática, por sua vez, é a evolução na distribuição do clima ao longo do tempo provocado, principalmente, por emissões antropogênicas de gases causadores do efeito estufa. 
Sobre as variações climáticas, há duas principais causas de variações: forma contínua, que influencia os fenômenos biológicos; e a forma episódica, por meio de eventos extremos (Confalonieri, 2003). Já as mudanças climáticas, segundo Nieuwolt e McGregor (1998) apud Mendonça (2003), tem origem em causas externas (mudanças na órbita dos planetas), fatores internos (mudanças na circulação oceânica, na composição de gases da atmosfera e nas condições da camada geográfica) e nas atividades humanas (queima de combustíveis fósseis, desmatamento, gases do efeito estufa).

Para o estudo da manifestação da dengue foram utilizados dados do banco de dados do SINANSistema Nacional de Acompanhamento e Notificação- do Ministério da Saúde, bem como dados fornecidos pela Secretaria Estadual de Saúde. Os dados meteorológicos serão fornecidos pelo INMET- Instituto Nacional de Meteorologia.

Foram escolhidos os 10 municípios com maior contingente populacional e que possuíssem estações meteorológicas: Amambaí, Aquidauana, Campo Grande, Corumbá, Dourados, Maracaju, Parnaíba, Ponta Porã, Sidrolândia e Três Lagoas.O ano de 2009 foi escolhido por apresentar o maior surto de dengue desde os registros do SINAN.Os dados foram tabulados e trabalhados graficamente com o auxílio do software Microsoft Office, mais especificadamente, da planilha Excel versão 2003.

Em seguida ocorreu o estabelecimento de correlações entre a temperatura do ar, pluviosidade e número de casos de dengue. Realizaram-se dois grupos de correlação para cada dupla de variável correlacionada.

As correlações foram realizadas por meio do software SPSS 9.0 (StatisticalPackage for the Social Sciences), onde $r<1$ representa uma perfeita correlação negativa e $r>1$ é perfeita correlação positiva. $\mathrm{O} r$ de Pearson assume apenas valores entre -1 e 1. Este software permite diferentes análises de dados, teste de hipóteses e criação de modelos de projeção. O método estatístico utilizado foi a "correlação de Pearson" que por meio de seu coeficiente pode-se estimar a força da relação entre duas variáveis.

A fórmula do coeficiente de correlação de Pearson é então,

$$
r=\frac{\sum\left(x_{i}-\bar{x}\right)\left(y_{i}-\bar{y}\right)}{\sqrt{\left(\sum\left(x_{i}-\bar{x}\right)^{2}\right)\left(\sum\left(y_{i}-\bar{y}\right)^{2}\right)}}
$$

Fonte: LEG-UFPR 


\begin{tabular}{|l|r|r|r|}
\hline Colunas1 & Pluviosidade total & Temperatura média & Dengue \\
\hline Aquidauana & 1400,1 & 25,06 & 332 \\
\hline Campo Grande & 1689,9 & 23,47 & 2725 \\
\hline Dourados & 1532,9 & 22,81 & 848 \\
\hline Três Lagoas & 1350 & 24 & 0 \\
\hline Corumba & 421,4 & 26,84 & 5767 \\
\hline Ponta Porã & 1809,2 & 21,47 & 140 \\
\hline Sidrolândia & 1384,4 & 23,34 & 9 \\
\hline Maracaju & 914,7 & 22,93 & 70 \\
\hline Parnaíba & 1284,22 & 23,89 & 94 \\
\hline Amambaí & 1405,9 & 21,78 & 3 \\
\hline
\end{tabular}

Tabela 1: Dados climatológicos e da dengue nos municípios escolhidos

Fonte: INMET e DATASUS

\section{3 - Resultados}

No ano estudado foram notificados 9922 casos nos 10 municípios, porém com grande diferença na distribuição. Corumbá obteve mais da metade dos casos, enquanto Três Lagoas não teve nenhum caso notificado em 2009.

O ano escolhido apresentou chuvas atípicas acima do esperado, somando-se as 10 cidades, a pluviosidade total é de $13192,72 \mathrm{~mm}$, porém as temperaturas médias seguiram no padrão esperado. O aumento da pluviosidade em 2009 alertou a Secretária da Saúde sobre os possíveis casos de dengue.

Foi observado que no ano estudado, o mês de abril apresentou maior quantidade de casos nos municípios, enquanto os meses de julho, agosto e setembro apresentavam casos reduzidos ou nenhum caso. Isso indica que nestes períodos mecanismos como criadouros artificiais contribuíram para proliferação da doença mesmo em meses considerados não endêmicos.

Observa-se que nos meses que mais tiveram casos registrados, as temperaturas são significativamente mais altas que aos demais meses, sugerindo a temperatura ser um fator importante para o início das epidemias. 


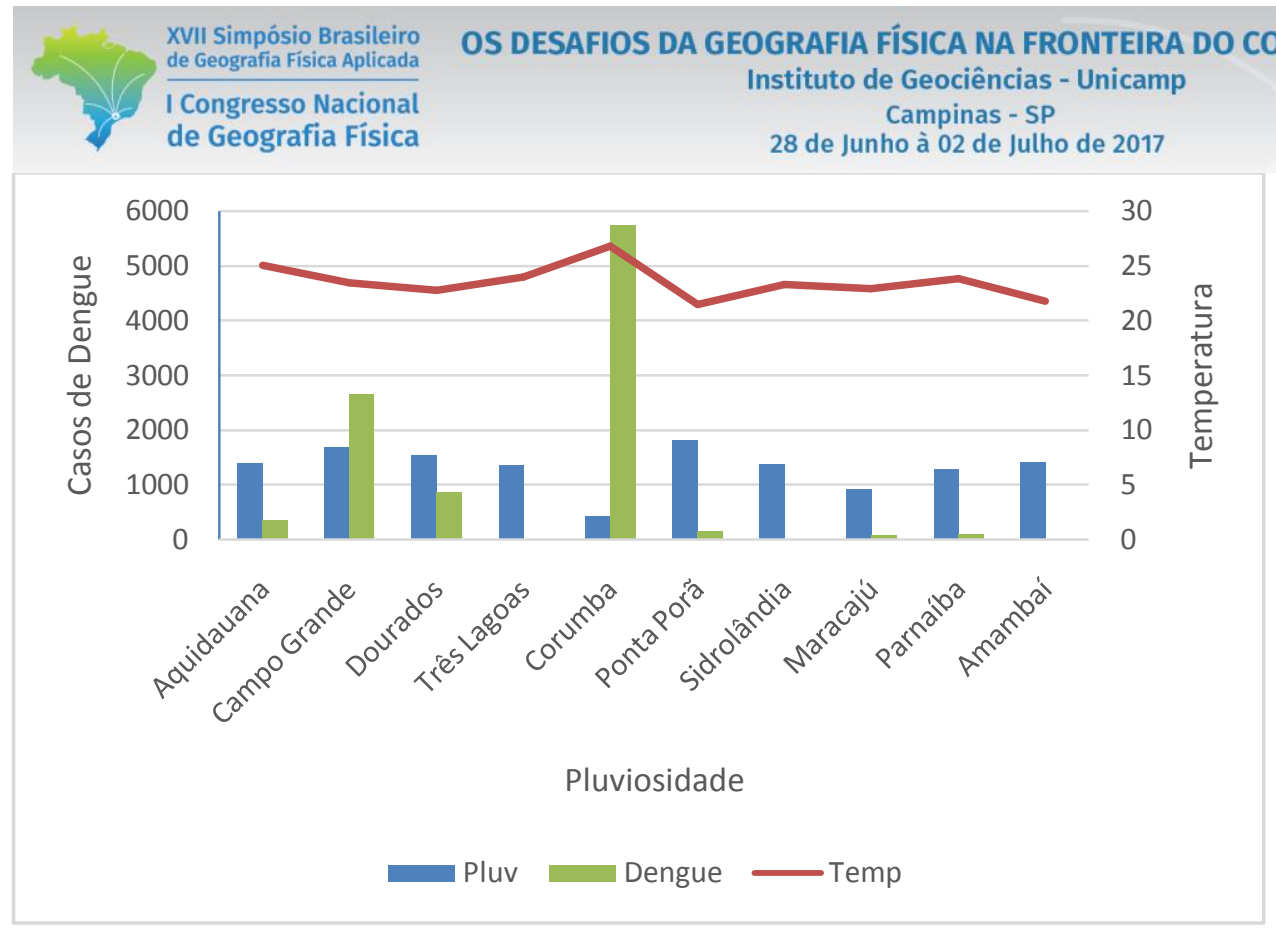

Figura 3: Gráfico dengue x pluviosidade x temperatura

Os resultados de correlação entre os casos de dengue e variáveis climáticas encontram-se na Tabela 2. Não foi observada associação entre os valores de temperatura média mensal e número de casos do mesmo mês de análise. A cidade de Aquidauana apresentou uma correlação positiva considerável quando a variável utilizada é a pluviosidade.

Na cidade de Três Lagoas não foi possível realizar a correlação devido ao número de casos da dengue ser nulo.

\begin{tabular}{|c|c|c|}
\hline Amambaí & Dengue vs. Temp & Dengue vs. Pluvi \\
\hline & $-0,385935573$ & $-0,425622045$ \\
\hline \multirow[t]{2}{*}{ Aquidauana } & Dengue vs. Temp & Dengue vs. Pluvi \\
\hline & 0,368611475 & 0,694620721 \\
\hline \multirow[t]{2}{*}{ Campo Grande } & Dengue vs. Temp & Dengue vs. Pluvi \\
\hline & 0,286276503 & 0,478093616 \\
\hline \multirow[t]{2}{*}{ Corumba } & Dengue vs. Temp & Dengue vs. Pluvi \\
\hline & 0,08344464 & $-0,305239807$ \\
\hline \multirow[t]{2}{*}{ Dourados } & Dengue vs. Temp & Dengue vs. Pluvi \\
\hline & 0,099380758 & $-0,303970895$ \\
\hline \multirow[t]{2}{*}{ Maracaju } & Dengue vs. Temp & Dengue vs. Pluvi \\
\hline & $-0,374982007$ & $-0,359251475$ \\
\hline Parnaíba & Dengue vs. Temp & Dengue vs. Pluvi \\
\hline
\end{tabular}




\begin{tabular}{|c|c|c|}
\hline Ponta Porã & Dengue vs. Temp & Dengue vs. Pluvi \\
\hline & 0,008183835 & $-0,247691067$ \\
\hline \multirow[t]{2}{*}{ Sidrolândia } & Dengue vs. Temp & Dengue vs. Pluvi \\
\hline & 0,468319228 & 0,149983379 \\
\hline
\end{tabular}

\section{4- Considerações Finais}

Mesmo sendo uma doença tipicamente sazonal são registrados casos da dengue tanto no período chuvoso quanto no seco, haja vista que a redução da densidade vetorial de adultos nos meses mais frios e secos não é suficiente para cessar a transmissão da doença.

Sem dúvidas, as maiores taxas de incidência de dengue em 2009 foi na zona de fronteira, onde ocorrem em áreas em que o ambiente é altamente propício a proliferação e circulação de vetores, porém somente o fator natural não é capaz de explicar a variabilidade da distribuição da dengue na região, para melhor compreensão dos surtos da doença é necessário compreender diversos elementos da dinâmica regional, como o povoamento e a mobilidade transfronteiriça. Apesar de conter um sentido ambíguo, a fronteira nos remete não só a ideia de soberania, mas também a ideai de integração de povos, compartilhando suas culturas e tradições remetendo a um cotidiano compartilhado que atravessa qualquer limite fronteiriço. Apesar de existir a ideia de que as regiões fronteiriças tem um "marco" de separação cultural por possuírem diferentes legislações e interesses, a fronteira é uma zona de compartilhamento.

Nesse aspecto podem-se citar os vetores causadores de doenças, que não reconhecem limites fronteiriços e acabam assim ampliando os limites das patologias, nesse sentido as ações de combate precisam ser desenvolvidas de maneira contínua e em ambos os lados. Os esforços de combate somente em um lado da fronteira não será suficiente para amenizar a vulnerabilidade e os riscos que estão suscetíveis às populações locais.

De fato a presente pesquisa abre-se para que estudos futuros sejam conduzidos no âmbito de inserir maiores quantidades de dados, porém evidência a necessidade de maior colaboração entre os países até mesmo na troca de dados dos agravos.

O estudo demonstrou a necessidade de dar continuidade ao estudo analisando não somente mais anos como também maior quantidade municípios do estado. Desde Hipócrates, acredita que as doenças apresentam causas ambientais, porém no caso da dengue, devemos considerar que a ausência de planejamento e a falta de políticas públicas eficientes passam a serem os principais fatores de contaminação. 
De acordo com Soares (2013), o estado do Mato Grosso do Sul possui poucas informações disponíveis sobre o descarte dos resíduos sólidos, evidenciando assim uma falha no planejamento. Outra questão citada é a de que não somente resíduos dispostos incorretamente são potenciais causadores de danos socioambientais, mas também quando acumulados nas residências podem causar danos à saúde pública, sobretudo, na proliferação de vetores capazes de transmitir doenças à população.

É sob essa perspectiva que essa pesquisa vêm sendo desenvolvida em nível de mestrado no Programa de Pós-Graduação em Geografia da UFGD, no âmbito do LGF (www.lgf.ggf.br), com o intuito de buscar compreender melhor a complexa relação com o número de casos de dengue no Mato Grosso do Sul, sua associação com os fatores climáticos, sem deixar de lado os condicionantes ambientais envolvidos na proliferação e ocorrência da dengue.

\section{5 - Referências}

BESANCENOT, J-P. Climat et santé.Paris: PUF, 2001. (Medicine et santé)

CALADO, D. C.; NAVARRO-SILVA, M. A. Influência da temperatura sobre a longevidade, fecundidade e atividade hematofágica de Aedes (Stegomyia) albopictusSkuse, 1894 (Diptera, Culicidae) sob condições de laboratório. Rev. Bras. Entomol., v. 46, n.1, p. 9398, 2002.

CONFALONIERI U, E. C. Variabilidade climática, vulnerabilidade social e saúde no Brasil. Terra Livre, São Paulo, v. I, n. 20, p193-204, jan/jul. 2003.

CONSOLI, R., OLIVEIRA, R. L. Principais mosquitos de importância sanitária no Brasil. FIOCRUZ, 1994. 228P

COSTA, M. A. R. A ocorrência do Aedes Aegypti na região Noroeste do Paraná: um estudo sobre a epidemia da dengue em Paranavaí - 1999, na perspectiva da geografia médica. Presidente Prudente (Dissertação de Mestrado em Geografia). FCT/UNESP, 2001.

DAJOZ, R. Ecologia geral. Petrópolis: Vozes, 1983. 472p.

JOHANSSON, M.A; CUMMINGS, D.A.T; GLASS, G.E. Multiyear Climate Variability and Dengue-El Niño Southern Oscillation, Weather, and Dengue Incidence in Puerto Rico, Mexico, and Thailand: A Longitudinal Data Analysis.PLoSMed 6(11): e1000168. doi:10.1371/journal.pmed.1000168, 2009.

MENDONÇA, F. A. Aquecimento global e saúde: uma perspectiva geográfica- notas introdutórias. Revista Terra Livre. São Paulo. Ano 19, v.1, n.20.2003, p. 205-221.

MEDONÇA, F. A.; PAULA, E. V.; OLIVEIRA, M. M.; JORGE, F.V.; PINTO, L. R; KRUGER, G. H. B. Clima e dengue: abordagem introdutória da evolução da dengue na região Sul do Brasil. In: VI Simpósio Brasileiro de Climatologia Geográfica, Aracaju/SE, 2004b. CD-ROM. 
MONDINI A, CHIARAVALLOTI-Neto F. Análise espacial da transmissão de dengue em cidade de porte médio do interior paulista.RevSaude Publica. 2007;39(3): 444-51.

ODUM, E. P. Ecologia. Rio de Janeiro: Guanabara, 1988.

OMS. Organização Mundial de Saúde. Dengue guidelines for diagnosis, treatment, prevention and control, new edition. Genebra: Organização Mundial de Saúde. 2009.

OPAS. Organização Pan-Americana de Saúde. Dengue and Dengue Haemorraghic Fever in the Americas: guidelines for prevention and control. Washington: Organização PanAmericana de Saúde. 1997.

OPAS. Organização Pan-Americana de Saúde. Dengue time line. Disponível em: www.paho.org/English/AD/DPC/CD/dengue_timeline.xls+DENGUE+TIMELINE,+XLS. 2001a, Acesso em: 5 out 13 .

PARRA, M. Regiões bioclimáticas do Estado do Mato Grosso do Sul, 2002. www.rc.unesp.br/igce/geografia/pos/download/2002/regioesbioclimaticas.pdf,acesso em 15 de abril de 2016.

SILVA, R. F.; NEVES, D. P. Os mosquitos (Diptera: Culicidae) do Campus Ecológico da UFMG, Belo Horizonte, Minas Gerais. Memórias do Instituto Oswaldo Cruz, v. 84, Supl. IV, p. 501-503, 1989.

SILVA, J. S. MARIANO, Z. De F. SCOPEL, I. A influencia do clima urbano na proliferação do mosquito Aedes Aegypti em Jataí(GO) na perspectiva da geografia médica.HygeiaRevista Brasileira de Geografia Médica e da Saúde, Uberlândia, v. 5, n 2, p. 33-46, Dez. 2007.

SILVA, I. A. Distribuição das chuvas e ocorrência de casos confirmados de dengue em Uberlândia-MG. In: SIMPÓSIO BRASILEIRO DE CLIMATOLOGIA GEOGRÁFICA: Climatologia e Gestão do Território, 9, 2010. Fortaleza-CE. Anais...Fortaleza-CE: ABclima e UFCE, 2010, p. 1- 9. (CD-ROM).

SIMMONS, C. P.; FARRAR, J. J.; CHAU, N. V. V.; WILLS, B. Dengue. The New England Journal of Medicine, v. 366, n. 15, p. 1423-1432, april 12, 2012.

SOARES, N. K.; Diagnóstico das áreas de destinação dos resíduos sólidos urbanos na bacia hidrográfica do rio Ivinhema / MS, 2013, 154f., Dissertação (Mestrado em Geografia), Instituto de Geografia, Universidade Federal da Grande Dourados, Dourados, 2013.

TORRES, E. M. Dengue y dengue hemorrágico. Buenos Aires/Argentina: Editora. Nacional da Universidade de Quilmes, 1998, p. 24-58.

ZAVATTINI, JA. As chuvas e as massas de ar no estado de Mato Grosso do Sul: estudo geográfico com vista à regionalização climática [online]. São Paulo: Editora UNESP; São Paulo: Cultura Acadêmica, 2009. 212 p. ISBN 978-85-7983-002-0. AvailablefromSciELO Books . 